・研究简报・

\title{
铋(III)苯乙酸-1,10-邻菲罗啉三元配合物 $\left[\mathrm{Bi}_{2}(\mathrm{PPA})_{6} \bullet(\mathrm{Phen})_{2}\right]$ 的合成、 晶体结构及抑菌活性
}

\author{
杨树平 $*, a$ 韩立军 $b$ 潘 燕 ${ }^{c}$ 吴争鸣 ${ }^{a}$ 何欣然 ${ }^{a}$ 陈丽娟 ${ }^{a}$ \\ $\left({ }^{a}\right.$ 淮海工学院化学工程学院 连云港 222005) \\ ( ${ }^{b}$ 淮海工学院数理学院 连云港 222005) \\ ( ${ }^{c}$ 北京大学医学部基础医学院药理系及国家天然仿生药物重点实验室 北京 100191)
}

\begin{abstract}
摘要 合成了铋 $(\mathrm{III})$ 苯乙酸-1,10-邻菲罗啉 $\left[\mathrm{Bi}_{2}(\mathrm{PPA})_{6} \cdot(\mathrm{Phen})_{2}\right]\left(\mathrm{HPPA}=\right.$ 苯乙酸 $\left(\mathrm{C}_{8} \mathrm{H}_{7} \mathrm{O}_{2}\right), \mathrm{Phen}=1,10$-邻菲罗啉 $\left.\left(\mathrm{C}_{12} \mathrm{H}_{8} \mathrm{~N}_{2}\right)\right)$ 三元配合物, 并通过元素分析和红外(IR)光谱对其进行了表征, 用单晶 $\mathrm{X}$-射线衍射测定了配合物的晶体结 构. 配合物属于三斜晶系, $P-1$ 空间群, $a=0.9100(6) \mathrm{nm}, b=1.2617(8) \mathrm{nm}, c=1.3324(9) \mathrm{nm}, \alpha=89.757(8)^{\circ}, \beta=$

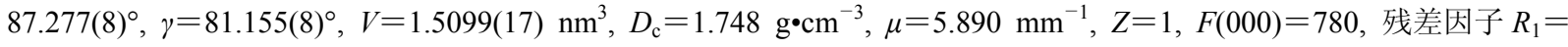
$0.0484, w R_{2}=0.1090[I>2 \sigma(I)], S=1.002$. 标题化合物为双核铋(III)配合物，金属中心 $\mathrm{Bi}(\mathrm{III})$ 原子与三个苯乙酸根 $(\mathrm{PPA})$ 中的 6 个氧原子、一个 1,10-邻菲罗啉分子中的两个氮原子和一个桥连氧原子进行配位, 形成九配位的扭曲三帽三方柱 配位十四面体, 围绕铋原子的价键总数 $V_{\mathrm{Bi}(1)}=2.897$, 两个 $\mathrm{Bi}(\mathrm{III})$ 原子通过两个桥连氧原子连结成中心对称的分子, $\mathrm{Bi} \cdots \mathrm{Bi}$ 间距离为 $0.4469(2) \mathrm{nm}$. 初步抑菌试验结果显示, 配合物对大肠杆菌 $(E . C o l i)$ 、金黄色葡萄球菌(S.Aureus)、枯草 芽孢杆菌(B.Subtilis)表现出相似且良好的抑菌效果.
\end{abstract}

关键词＼cjkstart铋(III)配合物; 晶体结构; 抗菌活性; 苯乙酸; 邻菲罗啉

\section{Synthesis, Crystal Structure and Antibacterial Activity of A Bis- muth(III) Complex $\left[\mathrm{Bi}_{2}(\mathrm{PPA})_{6} \bullet(\text { Phen })_{2}\right]$ with Phenylacetic Acid and 1,10-Phenanthroline}

\author{
Yang, Shuping ${ }^{*, a} \quad{\text { Han, } \text { Lijun }^{b} \quad \text { Pan, Yan }}^{c} \quad$ Wu, Zhenming ${ }^{a}$ \\ He, Xinran ${ }^{a} \quad$ Chen, Lijuan ${ }^{a}$ \\ ( ${ }^{a}$ School of Chemical Engineering, Huaihai Institute of Technology, Lianyungang 222005) \\ $\left({ }^{b}\right.$ School of Mathematics and Science, Huaihai Institute of Technology, Lianyungang 222005) \\ ( ${ }^{c}$ Department of Pharmacology, School of Basic Medical Sciences and State Key Laboratory of Natural and Biomimetic \\ Drugs, Peking University, Beijing 100191)
}

\begin{abstract}
A bismuth(III) complex $\left[\mathrm{Bi}_{2}(\mathrm{PPA})_{6} \cdot(\mathrm{Phen})_{2}\right] \quad(\mathrm{HPPA}=$ phenylacetic acid; Phen $=$ 1,10-phenanthroline) was synthesized and characterized by elemental analysis and infrared (IR) spectroscopy, its crystal structure was determined by single crystal X-ray diffraction. The crystal of bismuth(III) complex belongs to triclinic system with space group $P-1, a=0.9100(6) \mathrm{nm}, b=1.2617(8) \mathrm{nm}, c=$ $1.3324(9) \mathrm{nm}, \alpha=89.757(8)^{\circ}, \beta=87.277(8)^{\circ}, \gamma=81.155(8)^{\circ}, V=1.5099(17) \mathrm{nm}^{3}, D_{\mathrm{c}}=1.748 \mathrm{~g} \cdot \mathrm{cm}^{-3}, \mu=$ $5.890 \mathrm{~mm}^{-1}, Z=1, F(000)=780$, residual factors $R_{1}=0.0484, w R_{2}=0.1090[I>2 \sigma(I)], S=1.002$. The com-

\footnotetext{
*E-mail: spyang66381@yahoo.cn
}

Received September 10, 2011; revised November 18, 2011; accepted December 19, 2011.

Project supported by the National Natural Science Foundation of China (No. 30901815) and Natural Science Foundation of Huaihai Institute of Technology, China (No. Z2009019).

国家自然科学基金(No. 30901815)和淮海工学院自然科学基金(No. Z2009019)资助项目.
\end{abstract}


complex is a binuclear bismuth(III) compound, and every bismuth(III) atom is nine-coordinated by six $\mathrm{O}$ atoms from three ligands (PPA) and two $\mathrm{N}$ atoms from one ligand (Phen) and one bridged $\mathrm{O}$ atom, and the coordination polyhedron around the bismuth(III) atom can be best described as a distorted tricapped trigonal-prismatic tetrakaidecahedron geometry, the valence sum of around $\mathrm{Bi}$ atom $V_{\mathrm{Bi}(1)}=2.897$. The distance of $\mathrm{Bi} \cdots \mathrm{Bi}$ is $0.4469(2) \mathrm{nm}$. The complex has a good effect against E. Coli, S. Aureus and B. Subtilis.

Keywords bismuth(III) complex; crystal structure; antibacterial activity; phenylacetic acid; 1,10-phenanthroline

铋化合物作为药物已经有 200 多年的历史, 在治疗 各种微生物感染, 包括梅毒、腹泻、胃炎和结肠炎等方面 效果明显, 且毒性低. 近年来随着一些含铋药物如枸侬 酸铋 $(\mathrm{CBS})$ 、雷尼替丁枸橡酸铋 $(\mathrm{RBC})^{[1,2]}$ 、碱式水杨酸 铋 ${ }^{[3,4]}$ 和果胶铋 ${ }^{[5,6]}$ 相继用于治疗消化不良、溃疡性结肠 炎、胃溃疡等消化系统疾病, 使得铋配合物在医药领域潜 在应用的研究得到重视. 许多铋配合物相继被报道, 其 中一些铋配合物具有良好生物活性, 如抗菌 ${ }^{[7,8]}$ 、抗利什

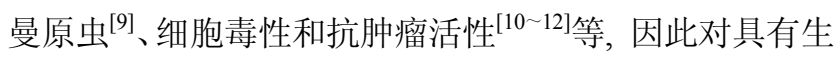
物活性铋配合物的研究在医药领域是一项有意义的工作.

金属铋(III)因具有较大的离子半径, 使得其有较强 的变形性和极化能力, 在形成配合物时可以克服空间位 阻, 接受多对电子而形成不同配位数的配合物, 配位数 可高达 $10^{[13]}$, 金属铋(III)极易与含有氧和氮原子的多齿 配体进行配位, 使形成的配合物具有结构多样性和广泛 的用途 ${ }^{[8,14]}$. 苯乙酸 (HPPA)作为配体具有良好的配位能 力, 根据 Pearson 的“硬-软酸-碱” 理论 ${ }^{[14]}$, 苯乙酸分子内 含有属于硬碱的羧基, 即能与金属螯合, 又能与金属桥 联, 形成动力学稳定的多核配合物 ${ }^{[15]}$. 1,10-邻菲罗啉 (Phen)是一类被广泛使用的 $N, N$-双齿配体, 在配位化学 中常常用于制备含混合配体的配合物, 混合配体的存在 常常使得配合物键合方式、光谱性质、配位环境的几何 构型和生物活性发生改变 ${ }^{[16]}$, 许多含有羒基和 1,10-邻菲 罗啉三元金属配合物被报道, 其中一些还表现出良好的 生物活性或其它用途 ${ }^{17 ~ 21]}$. 本文新合成了一个铋(III)苯 乙 酸 (HPPA)-1,10- 邻菲罗啉 (Phen) 三元配 合物 $\left[\mathrm{Bi}_{2}(\mathrm{PPA})_{6} \cdot(\mathrm{Phen})_{2}\right]$, 并通过元素分析和红外(IR)光谱对 其进行了表征, 用单晶 X-射线衍射测定了配合物的晶体 结构. 同时, 用琼脂扩散法初步研究了标题配合物对大 肠杆菌、金黄色葡萄球菌和枯草杆菌的抑菌活性, 为今后 金属铋配合物在生物无机化学、药物化学、分子生物学 等领域的研究提供了一些具有理论和应用价值的信息.

\section{1 实验部分}

\section{1 仪器和试剂}

Perkin Elmer-2400 型元素分析仪(美国 Perkin-Elmer
公司); WGH-30/6 型红外光谱仪(天津市港东科技发展 有限公司); pHS-25 型酸度计(上海精科雷磁仪器厂); Bruker-SMART-1000 单晶衍射仪(德国 Bruker 公司).

试剂均为分析纯, 购自国药集团上海化学试剂有限 公司, 使用前未经进一步纯化; 实验菌株: 大肠杆菌 $(E$. Coli)、金黄色葡萄球菌(S. Aureus) 和枯草芽狍杆菌(B. Subtilis)均由淮海工学院海洋学院微生物实验室提供; 实验用水为二次蒸馏水.

\section{2 标题配合物 $\left[\mathrm{Bi}_{2}(\mathrm{PPA})_{6} \bullet(\text { Phen })_{2}\right]$ 的合成}

将硝酸铋 $\left[\mathrm{Bi}\left(\mathrm{NO}_{3}\right)_{3} \cdot 5 \mathrm{H}_{2} \mathrm{O}\right] 0.485 \mathrm{~g}(1 \mathrm{mmo} 1), 95 \%$ 乙 醇 $10 \mathrm{~mL}$, 放入 $100 \mathrm{~mL}$ 三口烧瓶中, 置于 $65{ }^{\circ} \mathrm{C}$ 水浴中 加热搅拌, 然后逐滴加入 $25 \mathrm{~mL}$ 含有苯乙酸 $0.474 \mathrm{~g} \mathrm{(3}$

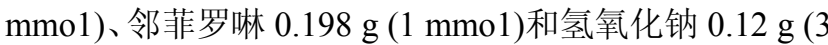
$\mathrm{mmo1})$ 的 $95 \%$ 乙醇溶液 $(\mathrm{pH}=6 \sim 7), 70{ }^{\circ} \mathrm{C}$ 下反应 $6 \mathrm{~h}$, 趁 热抽滤, 滤饼用少量水、乙醇洗涤, 自然干燥, 得粗品, 粗品用乙醇-DMF 混合溶剂 $(V: V=20: 1)$ 重结晶, 得适 合于单晶分析的浅黄色透明柱状晶体，产率约 $57 \%$ (以 $\mathrm{Bi}\left(\mathrm{NO}_{3}\right)_{3} \cdot 5 \mathrm{H}_{2} \mathrm{O}$ 计), IR ( $\mathrm{KBr}$ 压片): 3074, 1603, 1582, $1545,1517,1427,1403,1386,1007,847,719,540,415$ $\mathrm{cm}^{-1}$. Anal. calcd for $\left[\mathrm{Bi}_{2}\left(\mathrm{C}_{8} \mathrm{H}_{6} \mathrm{O}_{2}\right)_{6} \cdot\left(\mathrm{C}_{12} \mathrm{H}_{8} \mathrm{~N}_{2}\right)_{2}\right]:$ C 54.41, H 3.30, N 3.53; found C 54.78, H 2.92, N 3.65.

\section{3 标题配合物的晶体结构测定}

选取一粒尺寸大小为 $0.51 \mathrm{~mm} \times 0.35 \mathrm{~mm} \times 0.23 \mathrm{~mm}$ 的单晶, 置于 Bruker SMART CCD 1000 单晶衍射仪上, 采用单色 $\mathrm{MoK} \alpha(\lambda=0.71073 \AA)$ 辐射, 在 298(2) K 温度 下, 以 $\varphi-\omega$ 方式扫描, 在 $2.23 \leqslant \theta \leqslant 26.53^{\circ}$ 范围内共收 集到衍射点 11060 个, 其中独立衍射点 5248 个 $\left(R_{\text {int }}=\right.$ $0.0653)$, 强度大于二倍平均背景强度 $[I>2 \sigma(I)]$ 的衍射 点 3824 个, 全部 5248 个独立衍射点被用于结构精修, 采用帕特森(Patterson)法确定了铋原子的坐标位置, 且 轮番使用 Fourier 合成确认其余非氢原子，氢原子的坐 标采用几何加氢法得到, 所有非氢原子被各向异性地精 修, 全部氢原子采用刚性模型处理. 结构分析表明, 配 合物晶体属于三斜晶系, $P-1$ 空间群, 晶胞参数: $a=$ 0.9100(6) nm, $b=1.2617(8) \mathrm{nm}, c=1.3324(9) \mathrm{nm}, \alpha=$ $89.757(8)^{\circ}, \beta=87.277(8)^{\circ}, \gamma=81.155(8)^{\circ}, V=1.5099(17)$ 
$\mathrm{nm}^{3}, Z=1, D_{\mathrm{c}}=1.748 \mathrm{~g} \cdot \mathrm{cm}^{-3}, F(000)=780$, 吸收系数 $\mu$ $=5.890 \mathrm{~mm}^{-1}$, 残差因子 $R_{1}=0.0484, w R_{2}=0.1090[I>$ $2 \sigma(I)], S=1.002$. 最终差值电子云图中最大残余正峰和 洞分别为 $\Delta \rho_{\max }=2.915 \times 10^{3} \mathrm{e} \mathrm{nm}^{-3}$ 和 $\Delta \rho_{\min }=-1.893$ $\times 10^{3} \mathrm{e} \bullet \mathrm{nm}^{-3}$. 晶体结构解析和精修采用 SHELXS-97 和 SHELXL-97 程序完成 ${ }^{[22]}$, 晶体结构做图采用 Diamond 软件 ${ }^{[23]}, \mathrm{CCDC}: 827584$.

\section{4 抑菌试验}

用牛津杯法 ${ }^{[24]}$ 测试标题配合物对大肠杆菌、金黄色 葡萄球菌和枯草芽狍杆菌的抑菌活性. 实验采用体外活 性测试，首先将配体及硝酸铋用混合溶剂(DMSO- $\mathrm{H}_{2} \mathrm{O}$, $V: V=1: 5$ )配成浓度为 $5 \times 10^{-3} \mathrm{~mol} \cdot \mathrm{L}^{-1}$ 的溶液, 铋配 合物用混合溶剂(DMSO- $\mathrm{H}_{2} \mathrm{O}, V: V=1: 5$ )配成 5 个浓 度的梯度: 分别含药为 $5 \times 10^{-3}, 2.5 \times 10^{-3}, 1 \times 10^{-3}, 7.5$ $\times 10^{-4}$ 和 $5 \times 10^{-4} \mathrm{~mol} \cdot \mathrm{L}^{-1}$ 的溶液; 然后将受试菌均匀地 涂在普通琼脂培养基(牛肉膏、蛋白胨、氯化钠、琼脂 $=1: 2: 1: 4, \mathrm{pH}=7.0$ )平板上, 将已灭菌的直径为 8 $\mathrm{mm}$ 的牛津杯放入培养基平板上, 再将配体、硝酸铋溶 液及不同浓度的待测药液各 $100 \mu \mathrm{L}$ 分别放入平板之上 的牛津杯中, 在 $37{ }^{\circ} \mathrm{C}$ 温度下培养 $24 \mathrm{~h}$ 后观察并测量抑 菌圈直径 $(\mathrm{mm})$ 大小, 每个试样均平行测试 3 次, 以平均 值作为最后的实验结果.

\section{2 结果与讨论}

\section{1 配合物的红外光谱表征}

红外图谱显示, 在配合物红外光谱图中, 自由配体 苯乙酸中羧酸的 3 个特征吸收峰 $\left(v_{\mathrm{O}-\mathrm{H}}, v_{\mathrm{c}}=\mathrm{o}, \delta_{\mathrm{O}-\mathrm{H}}\right.$, $3203 \sim 2550 \mathrm{~cm}^{-1}, 1700 \mathrm{~cm}^{-1}, 933 \mathrm{~cm}^{-1}$ )均已消失, 同时 在 1545,1405 和 $1386 \mathrm{~cm}^{-1}$ 处出现三个新的吸收峰, 分 别归属于苯乙酸根 $\left(\mathrm{COO}^{-}\right)$的反对称伸缩振动吸收峰 $\left(v_{\mathrm{asCOO}}{ }^{-}, 1545 \mathrm{~cm}^{-1}\right)$ 和对称伸缩振动吸收峰 $\left(v_{\mathrm{a}(\mathrm{COO}}{ }^{-}\right)$, 1403 和 $\left.1386 \mathrm{~cm}^{-1}\right)$, 与自由配体苯乙酸 $\left(v_{\mathrm{as}\left(\mathrm{COO}^{-}\right)}=1700\right.$ $\mathrm{cm}^{-1}, v_{\mathrm{aCOO}}-=1410 \mathrm{~cm}^{-1}, \Delta v=290 \mathrm{~cm}^{-1}$ )相比, 发生了 明显的移动, 其 $\Delta v\left[v_{\mathrm{as}\left(\mathrm{COO}^{-}\right)}-v_{\mathrm{a}\left(\mathrm{COO}^{-}\right)}\right]$分别为 142 和 $169 \mathrm{~cm}^{-1}$, 大于 $100 \mathrm{~cm}^{-1}$, 而小于 $200 \mathrm{~cm}^{-1}$, 说明配体 的羧基根 $\left(\mathrm{COO}^{-}\right)$与中心金属 $\mathrm{Bi}(\mathrm{III})$ 原子可能存在 2 种配 位方式: 双齿和桥联 ${ }^{[25,26]}$. 自由配体邻菲罗啉(Phen)的 特征吸收峰(特征伸缩振动峰 $v_{\mathrm{C}=\mathrm{N}}, 1560 \mathrm{~cm}^{-1}$, 面外变 角振动 $\delta_{\mathrm{C}-\mathrm{H}}, 853,739 \mathrm{~cm}^{-1}$ ) 也发生了移动, 分别红移至 1517,847 和 $719 \mathrm{~cm}^{-1}$, 表明邻菲罗啉中的两个氮原子同

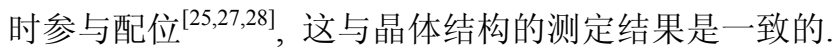
另外, 配合物在 $540,415 \mathrm{~cm}^{-1}$ 处出现了 2 个弱的吸收 峰, 分别归属于 $\mathrm{Bi}-\mathrm{N}$ 的伸缩振动峰 $v_{\mathrm{Bi}-\mathrm{N}}$ 和 $\mathrm{Bi}-\mathrm{O}$ 的 伸缩振动峰 $v_{\mathrm{Bi}-} \mathrm{O}^{[29,30]}, \mathrm{Bi}-\mathrm{O}$ 键和 $\mathrm{Bi}-\mathrm{N}$ 键吸收峰的出
现也进一步证实了新的铋配合物被合成.

\section{2 配合物的晶体结构描述}

标题配合物 $\left[\mathrm{Bi}_{2}(\mathrm{PPA})_{6} \cdot(\mathrm{Phen})_{2}\right]$ 的分子结构是一个 中心对称的双核化合物，如图 1 所示，主要键长与键角 列于表 1 , 配合物的不对称单元由一个铋(III)离子、一个 邻菲罗啉及三个配位形式不同的苯乙酸根阴离子组成.

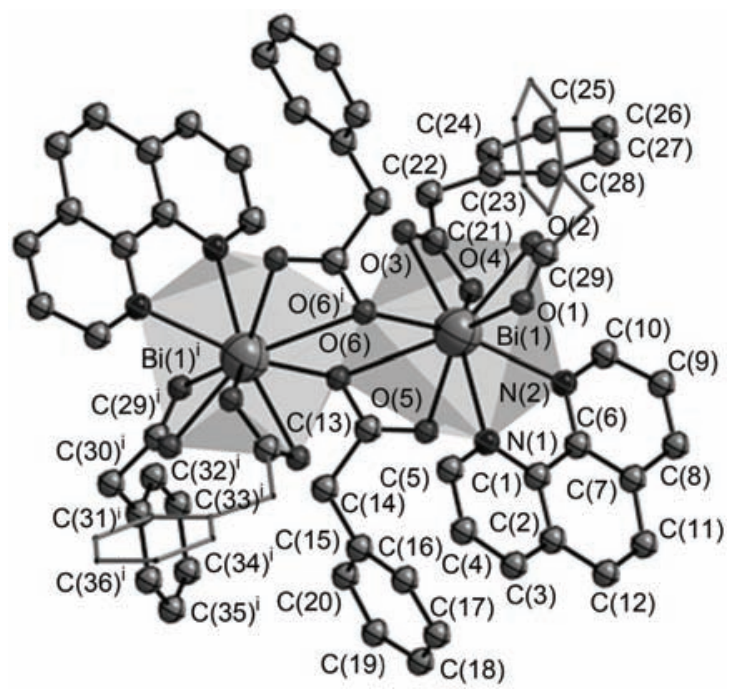

图 1 标题配合物 $\left[\mathrm{Bi}_{2}(\mathrm{PPA})_{6} \cdot(\mathrm{Phen})_{2}\right]$ 的分子结构和围绕金属 $\mathrm{Bi}(\mathrm{III})$ 原子的配位十四面体. 为了图形清楚部分原子标注在对 称位置[(i) $1-x, 1-y, 1-z]$, 并且省去了氢原子

Figure 1 Molecular structure of the title complex $\left[\mathrm{Bi}_{2}(\mathrm{PPA})_{6} \cdot\right.$ $\left.(\mathrm{Phen})_{2}\right]$ and the coordination tetrakaidecahedron geometry around $\mathrm{Bi}(\mathrm{III})$ atom. For clarity, the part of the atoms were labeled at symmetric position [(i) $1-x, 1-y, 1-z]$ and all $\mathrm{H}$ atoms have been omitted

由于金属铋最外层的 $\mathrm{p}, \mathrm{d}$ 轨道可以接受多对电子而 形成高配位数的配合物 ${ }^{[13]}$, 每个 $\mathrm{Bi}(1)$ 原子通过与来自 同一个邻菲罗琳分子中的两个氮原子 $[\mathrm{N}(1)$ 和 $\mathrm{N}(2)]$ 、三 个苯乙酸根阴离子中的六个氧原子 $[\mathrm{O}(1)-\mathrm{O}(6)]$ 及一个 来自对称位置的桥连氧原子 $\mathrm{O}(6)^{\mathrm{i}}$ [对称坐标: (i) $1-x, 1$ $-y, 1-z$, 下同]进行配位，形成一个九配位的扭曲三帽 三方柱十四面体(图 2). Bi(III) 原子处在三个帽顶原子 $\mathrm{O}(2), \mathrm{O}(5)$ 和 $\mathrm{O}(6)^{\mathrm{i}}$ 组成的赤道平面内, 其偏差为 0.0084(4) nm, 三方柱上、下底面分别由 $\mathrm{O}(3), \mathrm{O}(4), \mathrm{O}(6)$ 和 $\mathrm{O}(1), \mathrm{N}(1), \mathrm{N}(2)$ 组成，二者之间的夹角为 $27.5(3)^{\circ}$, 与 赤道平面的夹角分别为 23.7(2)和 25.2(2) ${ }^{\circ}$, 两个半分子 中的 $\mathrm{Bi}(1)$ 和 $\mathrm{Bi}(1)^{\mathrm{i}}$ 原子通过一对桥连氧原子 $\mathrm{O}(6)$ 和 $\mathrm{O}(6)^{\mathrm{i}}$ 连接, 将两个倒反联系的半分子连接成为一个中 心对称的双核配合物分子，对称中心在(1/2，1/2，1/2), 倒反配位多面体生成共享一条帽棱 $\left[\mathrm{O}(6) \cdots \mathrm{O}(6)^{\mathrm{i}}\right]$ 的双三帽三方柱配位十四面体几何构型(如图 1 所示), $\operatorname{Bi}(1) \cdots \operatorname{Bi}(1)$ 间距离为 $0.4469(2) \mathrm{nm}$, 这个值略小于两个 
Bi 原子的范德华半径和 $(0.468 \mathrm{~nm})$, 表明原子 $\mathrm{Bi}(1)$ 和 $\mathrm{Bi}(1)^{\mathrm{i}}$ 之间存在相互作用.

在配合物的晶体结构中, $\mathrm{Bi}-\mathrm{N}$ 键距为 $0.2613(8)$ 和 $0.2476(7) \mathrm{nm}, \mathrm{Bi}-\mathrm{O}$ 键的距离在 0.2347(6)到 0.2746(7) $\mathrm{nm}$ 之间, $\mathrm{Bi}-\mathrm{O}$ 键距有一个较大的范围. 为了对围绕着 Bi(III) 原子周围的配位环境及配位键的强弱有一个进一 步的认识, 利用两个相邻原子 $i$ 与原子 $j$ 之间成键的键价 数计算公式 ${ }^{[31]}: V_{i j}=\exp \left[\left(R_{i j}-d_{i j}\right) / b_{i j}\right]$ 和围绕 $i$ 原子总化 合价公式: $V_{i}=\sum_{j} V_{i j}$, 可以计算配合物中 $\mathrm{Bi}(\mathrm{III})$ 原子(原子 i) 与其周围每个配位的 $\mathrm{N}, \mathrm{O}$ 原子(原子 $j$ )间的配位键价 数 $\left(V_{i j}\right)$ 以及围绕着 $\mathrm{Bi}(\mathrm{III})$ 原子 $(i$ 原子 $)$ 的各个配位键的键 价数总和 $\left(V_{i}\right)$, 式中, $R_{i j}$ 为 $i$ 原子和 $j$ 原子单键键长、 $d_{i j}$ 为晶体学数据中 $i$ 原子和 $j$ 原子之间的键距、 $b_{i j}$ 是与 $i$ 和 $j$ 两个原子性质相关的参数 ${ }^{[32]}$. 此处, $R_{\mathrm{Bi}-\mathrm{N}}=0.202$ $\mathrm{nm} 、 b_{\mathrm{Bi}-\mathrm{N}}=0.035 \mathrm{~nm} ; R_{\mathrm{Bi}-\mathrm{O}}=0.2094 \mathrm{~nm} 、 b_{\mathrm{Bi}-\mathrm{O}}=0.037$ $\mathrm{nm}^{[33]}$, 对标题配合物的计算结果如下: $V_{\mathrm{Bi}(1)-\mathrm{N}(1)}=$ $0.246, \quad V_{\mathrm{Bi}(1)-\mathrm{N}(2)}=0.356, \quad V_{\mathrm{Bi}(1)-\mathrm{O}(1)}=0.278, \quad V_{\mathrm{Bi}(1)-\mathrm{O}(2)}=$ $0.454, V_{\mathrm{Bi}(1)-\mathrm{O}(3)}=0.319, \quad V_{\mathrm{Bi}(1)-\mathrm{O}(4)}=0.505, \quad V_{\mathrm{Bi}(1)-\mathrm{O}(5)}=$ $0.301, V_{\mathrm{Bi}(1)-\mathrm{O}(6)}=0.172, V_{\mathrm{Bi}(1)-\mathrm{O}(6)}{ }^{\mathrm{i}}=0.266$, 九配位的 $\mathrm{Bi}$ 原子总键价数 $V_{\mathrm{Bi}(1)}=2.897$, 这个值略小于 $\mathrm{Bi}(\mathrm{III})$ 离子 的化合价数 3, 也表明原子 $\mathrm{Bi}(1)$ 和 $\mathrm{Bi}(1)^{\mathrm{i}}$ 之间存在相互 作用. 按照文献 ${ }^{[34]}$ 中提出的建议: $\mathrm{Sb}-\mathrm{O}=0.26 \mathrm{~nm}$ 作为 锑氧键合的强键与次级键的分界. 这里换算为键价数 $V_{\mathrm{Sb}-\mathrm{O}}=0.184$, 与上面的计算结果比较, 得知 $\mathrm{Bi}(1)-$ $\mathrm{O}(6)=0.2746(7) \mathrm{nm}$ 是次级键, 其余可视为强键.

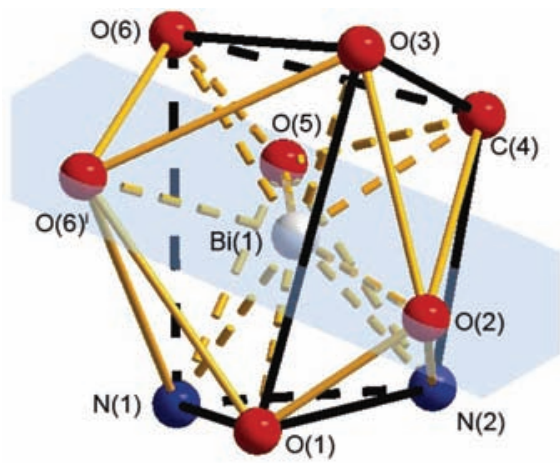

图 2 围绕 $\mathrm{Bi}(\mathrm{III})$ 原子的九配位扭曲三帽三方柱十四面体[对 称坐标: (i) $1-x, 1-y, 1-z]$

Figure 2 A distorted nine coordination tricapped trigonalprismatic tetrakaidecahedron around $\mathrm{Bi}(\mathrm{III})$ atom [Symmetry code: (i) $1-x, 1-y, 1-z]$

在配合物中, 羧基以两种配位方式和 $\mathrm{Bi}(\mathrm{III})$ 离子配 位: 即双齿螯合和桥连, $\mathrm{C}-\mathrm{O}$ 键的距离范围从 0.1210 (12)到 0.1263(11) $\mathrm{nm}$, 与原配体中羧基 $(\mathrm{COOH})$ 及 羧酸酯的 $\mathrm{C}-\mathrm{O}$ 键长 $[0.1205(3) \text { 和 } 0.1298(3) \mathrm{nm}]^{[35]}$ 相 比是趋中的，表明配合物中羒基与金属原子配位过程中
被质子化，这与配合物的红外图谱数据是一致的，因为 在红外图谱中 $1700 \mathrm{~cm}^{-1}$ 附近没有观察到 $\mathrm{COOH}$ 的特征 吸收峰.

在标题配合物的晶体结构中, 短距离的 $\pi \cdots \pi$ 相互作 用和 $\mathrm{C}-\mathrm{H} \cdots \pi$ 氢键连接分子形成二维的结构. 中心在 $(1 / 2,1 / 2,1 / 2)$ 的分子坐标在 $(x, y, z)$ 的配体邻菲罗琳上的 环 $\mathrm{N}(2) / \mathrm{C}(6)-\mathrm{C}(10)$ 的质心 $\mathrm{Cg}(1)$ 对着中心在 $(1 / 2,3 / 2$, 1/2)的分子上环质心 $\operatorname{Cg}(1)^{\mathrm{ii}}$ [对称坐标: (ii) $1-x, 2-y, 1$ $-z$,下同] 通过连续倒反和平移操作连结分子形成 $[01$ 0] 链(图 3), 其中环质心 $\mathrm{Cg}(1)$ 与 $\mathrm{Cg}(1)^{\mathrm{ii}}$ 之间的距离是 0.34703(14) $\mathrm{nm}$, 两个环面是平行的, 质心连线与环面 法线的夹角是 $20.82^{\circ}$.

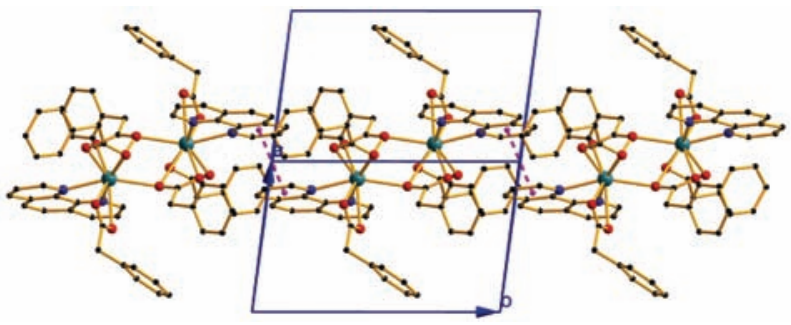

图 $3 \quad \pi \cdots \pi$ 相互作用连结的 $\left[\begin{array}{lll}0 & 1 & 0\end{array}\right]$ 链, 为了图形的清楚, 省掉 了氢原子

Figure 3 A chain by connected $\pi \cdots \pi$ interaction in the $\left[\begin{array}{lll}0 & 1 & 0\end{array}\right]$ direction. All $\mathrm{H}$ atoms have been omitted for clarity

中心在 $(1 / 2,1 / 2,1 / 2)$ 的分子坐标在 $(x, y, z)$ 的原子 $\mathrm{C}(24)$ 通过 $\mathrm{H}(24)$ 连结到中心在 $(-1 / 2,1 / 2,1 / 2)$ 分子上的 环质心 $\operatorname{Cg}(1)^{\mathrm{iii}}$ [对称坐标: (iii) $-1+x, y, z$ 下同], 通 过连续倒反和平移操作形成 $\left[\begin{array}{lll}1 & 0 & 0\end{array}\right]$ 分子链(图 4 ), 其 中 $\mathrm{C}(24) \cdots \mathrm{Cg}(1)^{\mathrm{iii}}=0.3476(11) \mathrm{nm}, \angle \mathrm{C}(24)-\mathrm{H}(24) \cdots$ $\operatorname{Cg}(1)^{\mathrm{iii}}=134.7^{\circ}$. [ [ $\left.\begin{array}{lll}0 & 1 & 0\end{array}\right]$ 和 $\left[\begin{array}{lll}1 & 0 & 0\end{array}\right]$ 分子链的结合产生 一个 $\left[\begin{array}{lll}0 & 0 & 1\end{array}\right]$ 的二维结构, 相邻的二维结构之间不存在 更短距离的 $\pi \cdots \pi$ 和 $\mathrm{C}-\mathrm{H} \cdots \pi$ 相互作用.

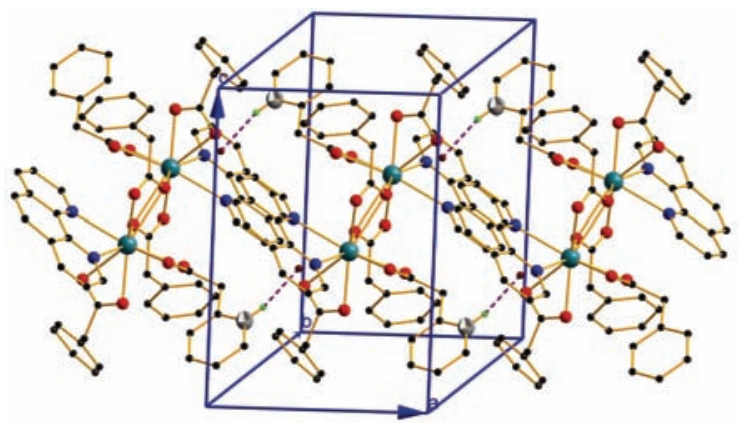

图 $4 \mathrm{C}-\mathrm{H} \cdots \pi$ 相互作用连结的 $\left[\begin{array}{lll}1 & 0 & 0\end{array}\right]$ 链, 为了图形的清楚省 掉了与氢键无关的氢原子

Figure $4 \mathrm{C}-\mathrm{H} \cdots \pi$ hydrogen-bonded chain in the $\left[\begin{array}{lll}1 & 0 & 0\end{array}\right]$ direction, $\mathrm{H}$ atoms not involved in the hydrogen bonds have been omitted for clarity 
表 1 标题配合物的主要键长 $(\mathrm{nm})$ 和键角 $\left(^{\circ}\right)$

Table 1 Selected bond lengths $(\mathrm{nm})$ and angles $\left({ }^{\circ}\right)$ of the title complex

\begin{tabular}{|c|c|c|c|}
\hline $\mathrm{Bi}(1)-\mathrm{O}(1)$ & $0.2567(8)$ & $\mathrm{Bi}(1)-\mathrm{O}(2)$ & $89(8)$ \\
\hline $\mathrm{Bi}(1)-\mathrm{O}(3)$ & $0.2517(7)$ & $\mathrm{Bi}(1)-\mathrm{O}(4)$ & $0.2346(7)$ \\
\hline $3 \mathrm{i}(1)-\mathrm{O}(5)$ & $0.2538(7)$ & $\mathrm{Bi}(1)-\mathrm{O}(6)$ & $0.2747(7)$ \\
\hline $\mathrm{i}(1)-\mathrm{O}(6)^{\mathrm{i}}$ & $0.2584(6)$ & & \\
\hline $\mathrm{Bi}(1)-\mathrm{N}(1)$ & $0.2614(8)$ & $\mathrm{Bi}(1)-\mathrm{N}(2)$ & $0.2474(7)$ \\
\hline $\mathrm{O}(1)-\mathrm{C}(29)$ & $0.1264(12)$ & $\mathrm{O}(2)-\mathrm{C}(29)$ & $0.1210(12)$ \\
\hline$(3)-C(21)$ & $0.1250(12)$ & $\mathrm{O}(4)-\mathrm{C}(21)$ & $0.1257(12$ \\
\hline$O(5)-C(13)$ & $0.1217(11)$ & $\mathrm{O}(6)-\mathrm{C}(13)$ & $0.1260(11)$ \\
\hline $\mathrm{N}(1)-\mathrm{C}(1)$ & $0.1338(12)$ & $\mathrm{N}(1)-\mathrm{C}(5)$ & 0.1 \\
\hline $\mathrm{N}(2)-\mathrm{C}(6)$ & $0.1339(12)$ & $\mathrm{N}(2)-\mathrm{C}(10)$ & $0.1334(11$ \\
\hline $\mathrm{O}(4)-\mathrm{Bi}(1)-\mathrm{O}(2)$ & $77.4(3)$ & $\mathrm{O}(4)-\mathrm{Bi}(1)-\mathrm{O}(3)$ & $53.0(2)$ \\
\hline $\mathrm{O}(2)-\mathrm{Bi}(1)-\mathrm{O}(3)$ & $73.4(3)$ & $\mathrm{O}(4)-\mathrm{Bi}(1)-\mathrm{O}(5)$ & 78. \\
\hline $\mathrm{O}(2)-\mathrm{Bi}(1)-\mathrm{O}(5)$ & $146.7(2)$ & $\mathrm{O}(3)-\mathrm{Bi}(1)-\mathrm{O}(5)$ & $108.2(3)$ \\
\hline $\mathrm{O}(4)-\mathrm{Bi}(1)-\mathrm{O}(1)$ & $128.1(2)$ & $\mathrm{O}(2)-\mathrm{Bi}(1)-\mathrm{O}(1)$ & $51.5(2)$ \\
\hline $\mathrm{O}(3)-\mathrm{Bi}(1)-\mathrm{O}(1)$ & 7 & $\mathrm{O}(5)-\mathrm{Bi}(1)-\mathrm{O}(1)$ & $141.3(3)$ \\
\hline $\mathrm{O}(4)-\mathrm{Bi}(1)-\mathrm{O}(6)^{\mathrm{i}}$ & $129.2(2)$ & $\mathrm{O}(2)-\mathrm{Bi}(1)-\mathrm{O}(6)^{\mathrm{i}}$ & $105.2(2)$ \\
\hline $\mathrm{O}(3)-\mathrm{Bi}(1)-\mathrm{O}(6)^{\mathrm{i}}$ & $78.6(2)$ & $\mathrm{O}(5)-\mathrm{Bi}(1)-\mathrm{O}(6)^{\mathrm{i}}$ & $107.7(2)$ \\
\hline $\mathrm{O}(1)-\mathrm{Bi}(1)-\mathrm{O}(6)^{\mathrm{i}}$ & $78.8(2)$ & $\mathrm{O}(4)-\mathrm{Bi}(1)-\mathrm{O}(6)$ & $86.3(2)$ \\
\hline $\mathrm{O}(2)-\mathrm{Bi}(1)-\mathrm{O}(6)$ & $149.7(2)$ & $\mathrm{O}(3)-\mathrm{Bi}(1)-\mathrm{O}(6)$ & $76.4(2)$ \\
\hline $\mathrm{O}(5)-\mathrm{Bi}(1)-\mathrm{O}(6)$ & $48.5(2)$ & $\mathrm{O}(1)-\mathrm{Bi}(1)-\mathrm{O}(6)$ & $142.4(2)$ \\
\hline \multicolumn{4}{|c|}{$\mathrm{O}(6)^{\# 1}-\mathrm{Bi}(1)-\mathrm{O}(6) 66.1(3)$} \\
\hline $\mathrm{O}(4)-\mathrm{Bi}(1)-\mathrm{N}(2)$ & $79.5(2)$ & $\mathrm{O}(2)-\mathrm{Bi}(1)-\mathrm{N}(2)$ & $77.8(2)$ \\
\hline $\mathrm{N}(2)-\mathrm{Bi}(1)-\mathrm{O}(3)$ & $128.3(2)$ & $\mathrm{N}(2)-\mathrm{Bi}(1)-\mathrm{O}(5)$ & $75.9(2)$ \\
\hline $\mathrm{N}(2)-\mathrm{Bi}(1)-\mathrm{O}(1)$ & $81.5(3)$ & $\mathrm{N}(2)-\mathrm{Bi}(1)-\mathrm{O}(6)^{\mathrm{i}}$ & $151.3(3)$ \\
\hline $\mathrm{O}(4)-\mathrm{Bi}(1)-\mathrm{N}(1)$ & $135.8(2)$ & $\mathrm{O}(2)-\mathrm{Bi}(1)-\mathrm{N}(1)$ & $115.8(2)$ \\
\hline $\mathrm{N}(2)-\mathrm{Bi}(1)-\mathrm{N}(1)$ & $64.3(2)$ & $\mathrm{O}(3)-\mathrm{Bi}(1)-\mathrm{N}(1)$ & $167.0(2)$ \\
\hline $\mathrm{O}(5)-\mathrm{Bi}(1)-\mathrm{N}(1)$ & $69.7(2)$ & $\mathrm{O}(1)-\mathrm{Bi}(1)-\mathrm{N}(1)$ & $72.4(2)$ \\
\hline $\mathrm{O}(6)^{\# 1}-\mathrm{Bi}(1)-\mathrm{N}(1)$ & $89.7(2)$ & $\mathrm{N}(2)-\mathrm{Bi}(1)-\mathrm{O}(6)$ & $124.4(2)$ \\
\hline $\mathrm{N}(1)-\mathrm{Bi}(1)-\mathrm{O}(6)$ & $93.7(2)$ & & \\
\hline
\end{tabular}

Symmetry transformations used to generate equivalent atoms: (i) $1-x, 1-y$, $1-z$.

\section{3 抗菌活性}

根据《消毒技术规范》中对抑菌作用的判断：抑菌 圈直径大于 $20 \mathrm{~mm}$ 表示具有强抑菌效果, 抑菌圈在 10 20 mm 为中等抑菌, 抑菌圈小于 $10 \mathrm{~mm}$ 为弱抑 菌 ${ }^{[36]}$, 实验显示, 配合物对 3 种受试菌均有相似且较强 的抑制作用, 配合物的抑菌作用随着浓度的降低而减 弱，最低抑菌浓度为 $5 \times 10^{-4} \mathrm{~mol} \cdot \mathrm{L}^{-1}$ ，低于这个浓度配 合物基本没有抑菌能力. 由表 3 可知, 在同一测试浓度 $\left(5 \times 10^{-3} \mathrm{~mol} \cdot \mathrm{L}^{-1}\right)$ 下, 硝酸铋 $\left[\mathrm{Bi}\left(\mathrm{NO}_{3}\right)_{3} \cdot 5 \mathrm{H}_{2} \mathrm{O}\right]$ 和自由配 体苯乙酸无抑菌作用, 自由配体邻菲罗啉显示中等程度 的抑菌作用，而铋配合物显示有强的抑菌作用. 表明形
成配合物后，铋离子与邻菲罗啉、苯乙酸的协同作用显 著增强了抑菌效果，但是该铋配合物的抑菌活性要弱于 文献[7]报道的铋配合物.

表 2 配合物的抑菌圈 $(\mathrm{mm})$

Table 2 Antibacterial activities of the complex in vitro (mm)

\begin{tabular}{|c|c|c|c|c|}
\hline \multirow[b]{2}{*}{ Compd. } & \multirow[b]{2}{*}{$\begin{array}{l}\text { 测试液浓度/ } \\
\left(\mathrm{mol} \cdot \mathrm{L}^{-1}\right)\end{array}$} & \multicolumn{3}{|c|}{ Antimicrobial circle diameter $/ \mathrm{mm}$} \\
\hline & & $\begin{array}{l}\text { 大肠杆菌 } \\
\text { (E. coli) }\end{array}$ & $\begin{array}{l}\text { 金黄色葡 } \\
\text { 萄球菌 }(S . \\
\text { aureus })\end{array}$ & $\begin{array}{l}\text { 枯草芽孢杆菌 } \\
\text { (B. subtilis) }\end{array}$ \\
\hline & $5 \times 10^{-3}$ & 29 & 27 & 26 \\
\hline & $2.5 \times 10^{-3}$ & 25 & 24 & 22 \\
\hline 配合物 & $1 \times 10^{-3}$ & 17 & 15 & 17 \\
\hline & $7.5 \times 10^{-4}$ & 13 & 13 & 14 \\
\hline & $5 \times 10^{-4}$ & 8 & 8 & 8 \\
\hline $\begin{array}{l}\text { 邻菲罗 } \\
\text { 啉 }\end{array}$ & $5 \times 10^{-3}$ & 20 & 19 & 19 \\
\hline 苯乙酸 & $5 \times 10^{-3}$ & 0 & 0 & 0 \\
\hline 醋酸铋 & $5 \times 10^{-3}$ & 0 & 0 & 0 \\
\hline
\end{tabular}

\section{3 结论}

合成并测定了铋(III)苯乙酸-1,10-邻菲罗啉三元配 合物 $\left[\mathrm{Bi}_{2}(\mathrm{PPA})_{6} \bullet(\mathrm{Phen})_{2}\right]$ 的晶体结构, 为双核 $\mathrm{Bi}$ 配合 物, 晶体属于三斜晶系, 不对称单元由一个铋(III)离子、 一个邻菲罗啉及三个配位形式不同的苯乙酸根阴离子 组成. 中心金属 $\mathrm{Bi}(\mathrm{III})$ 离子与来自三个苯乙酸根的六氧 原子、一个邻菲罗啉分子的两氮原子和一个桥连氧原子 进行配位，形成九配位的扭曲三帽三方柱配位十四面 体. 抗菌试验结果显示，配合物对大肠杆菌 (E. coli)、金 色葡萄球菌 $(S$. aureus) 和枯草杆菌(B. subtilis)有相似的 良好的抑菌作用. 这些实验研究为铋(III)类配合物在药 物领域的开发和利用提供了一定的理论依据和可靠的 实验证据.

\section{References}

1 Beales, I. LP. BMC Gastroenterology 2001, 1, 7.

2 Yang, N.; Sun, H.-Z. Coord. Chem. Rev. 2007, 251(17 20), 2354.

3 Suarez, F. L.; Furne, J.; Stiehm, J.; Garten, C.; Levitt, M. D. Dig. Dis. Sci. 2000, 45(7), 1444.

4 Dante, F.-Q.; Eduardo, S.-L.; R. Bradley, S.; Raul, L.-B.; Silvana, S.-A.; Miguel, C.-S.; Eduardo, E.-M. N. Engl. J. Med. 1993, 328(23), 1653.

5 Nie, Y.; Li, Y.; Wu, H.; Sha, W.; Du, H.; Dai, S.; Wang, H.; Li, Q. Helicobacter 1999, 4(2), 128.

6 Kotani, T.; Nagai, D.; Asahi, K.; Suzuki, H.; Yamao, F.; Kataoka, N.; Yagura, T. Antimicrob. Agents Chemother. 2005, 49(7), 2729. 
7 Nomiya, K.; Sekino, K.; Ishikawa, M.; Honda, A.; Yokoyama, M.; Kasuga, N. C.; Yokoyama, H.; Nakano, S.; Onodera, K. J. Inorg. Biochem. 2004, 98(2), 601.

8 Murafuji, T.; Fujiwara, Y.; Yoshimatsu, D.; Miyakawa, I.; Migita, K.; Mikata, Y. Eur. J. Med. Chem. 2011, 46(2), 519.

9 Philip, C.; Andrews, R. F.; Peter, C.; Junk, L. K.; Ish Kumar, J. G. M. J. Inorg. Biochem. 2011, 105(3), 454.

10 Li, H.-Y.; Lai, C. S.; Wu, J. Z.; Paul, C.; Dick de Vos, H.; Tiekink, E. R. T. J. Inorg. Biochem. 2007, 101(5), 809.

11 Smith, K. A.; Deacon, G. B.; Jackson, W. R.; Tiekink, E. R. T.; Rainone, S.; Webster, L. K. Met.-Based Drugs 1998, 5(5), 295.

12 Wang, X.-Y.; Zhang, X.-M.; Lin, J.; Chen, J.-W.; Xu, Q.; Guo, Z.-J. J. Chem. Soc., Dalton Trans. 2003, 2379.

13 Zhang, Z.-P.; Zhong, G.-Q.; Jiang, Q.-Y. Prog. Chem. 2008, 20(9), 1315 (in Chinese).

(张志朋, 钟国清, 蒋琪英, 化学进展, 2008, 20(9), 1315.)

14 Stavila, V.; Davidovich, R. L.; Gulea, A.; Whitmire, K. H. Coord. Chem. Rev. 2006, 250, 2782.

15 Viossat, V.; Lemoine, P.; Dayan, E.; Dung, N.-H.; Viossat, B. J. Mol. Struct. 2005, 741(1 3), 45.

16 Gup, R.; Kırkan, B. Spectrochim. Acta, Part A 2006, 64(3), 809.

17 Xu, H.-H.; Gu, J.-M. Chin. J. Inorg. Chem. 1998, 14(2), 237 (in Chinese).

(徐号华, 顾建明, 无机化学学报, 1998, 14(2), 237.)

18 Jiang, Q.-Y.; Shen, J.; Zhong, G.-Q. Prog. Chem. 2006, 18(12), 1634 (in Chinese).

(蒋琪英, 沈娟, 钟国清, 化学进展, 2006, 18(12), 1634.)

19 Sun, X.-D.; Sun, Y. Chem. Res. Appl. 2009, 21(9), 1275 (in Chinese).

(孔晓朵, 孙怡, 化学研究与应用, 2009, 21(9), 1275.)

20 Li, W.; Li, C.-H.; Yang, Y.-Q.; Xie, H.-P. Chin. J. Inorg. Chem. 2009, 25(6), 1106 (in Chinese).

(李薇, 李昶红, 杨颖群, 谢和平, 无机化学学报, 2009, 25(6), 1106.)

21 Le, X.-Y.; Yang, G.; Lu, Q.-M. Chin. J. Inorg. Chem. 2002, 18(4), 427 (in Chinese).

(乐学义, 杨光, 卢其明, 无机化学学报, 2002, 18(4), 427.)

22 Sheldrick, G. M. Acta Cryst. A 2008, 64(1), 112.

23 Brandenburg, K. Diamond, Crystal Impact GbR, Bonn, Germany, 1999.

24 Liu, G.-Z. Micro-biology and the Detect of Micro-biology,
People's Medical Publish House, Beijing, 1987, p. 100 (in Chinese).

(刘恭植, 微生物学和微生物学检验, 人民卫生出版社, 北京, 1987, p. 100.)

25 Nakamoto, K. Infrared and Raman Spectra of Inorganic and Coordination Compounds, Trans. Huang, D. R.; Wang, R. Q. Chemical Industrial Press, Beijing, 1986, p. 235 (in Chinese).

(中本一雄, 无机和配位化合物的红外和拉曼光谱, 黄德 如, 汪仁庆译, 化学工业出版社, 北京, 1986, p. 235.)

26 Gao, S.; Zhang, Z.-Y.; Huo, L.-H.; Zhao, H.; Zhao, J.-G. Chin. J. Inorg. Chem. 2005, 21(5), 771 (in Chinese).

(高山, 张竹艳, 霍丽华, 赵辉, 赵经贵, 无机化学学报, 2005, 21(5), 771.)

27 Yu, Y.-Y.; Liu, J.-F.; Li, H.-Q.; Zhao, G.-L. Acta Phys.-Chim. Sin. 2010, 26(6), 1535 (in Chinese).

(余玉叶, 刘建风, 李花琼, 赵国良, 物理化学学报, 2010, 26(6), 1535.)

28 Ying, H.-D.; Wang, C.-H.; Xu, F.-Q. Chin. J. Inorg. Chem. 2004, 20(3), 345 (in Chinese).

(尹汉东, 王传华, 许福泉，无机化学学报，2004，20(3), 345.)

29 Zhong, G.-Q.; Chen, M.-J.; Jiang, Q.-Y.; Zhang, Z.-P. J. Inorg. Synth. Cryst. 2010, 39(1), 281 (in Chinese).

(钟国清，陈明剑，蒋琪英，张志朋，人工晶体学报，2010, 39(1), 281.)

30 Guo, Y.-C.; Zhuo, L.-H. Chem. Res. 2003, 14(2), 39 (in Chinese).

(郭应臣, 卓立宏, 化学研究, 2003, 14(2), 39.)

31 Brese, N. E.; O'Keeffe, M. Acta Cryst. B 1991, 47(2), 192.

32 Brown, I. D. The Chemical Bond in Inorganic Chemistry, Oxford University Press, Oxford, 2002.

33 Brown, I. D.; Altermatt, D. Acta Cryst. B 1985, 41(4), 244.

34 Hu, S.-Z.; Chen, M.-D.; Robertson, B. E. Acta Phys.-Chim. Sin. 2005, 21(6), 646 (in Chinese).

(胡盛志, 陈明旦, Robertson, B. E. 物理化学学报, 2005, 21(6), 646.)

35 Hodgson, D. J.; Asplund, R. O. Acta Cryst. C 1991, 47(9), 1986.

36 Xia, Q.-C.; Shen, Z.-H.; He, Q.-Z.; Xu, D.-F.; Peng, Z.-F.; Shao, C.-Y. Acta Chim. Sinica 2009, 67(16), 1843 (in Chinese).

(夏庆春, 沈智慧, 何其庄, 许东芳, 彭子飞, 邵超英, 化 学学报, 2009, 67(16), 1843.) 